\title{
On $\mathrm{G}_{\mathrm{m}}$-boosting and Cyclostationary noise Mechanisms in Low- Voltage CMOS Differential Colpitts VCOs
}

\author{
Adil Koukab, and Omid Talebi Amiri \\ Swiss Federal Institute of Technology in Lausanne (EPFL), 1015 Lausanne Switzerland \\ adil.koukab@epfl.ch
}

\begin{abstract}
This paper presents a theoretical study of CMOS differential Colpitts VCOs. The objective is to provide a deep understanding of the different mechanisms that impact the performances of these VCOs, namely the Gm-boosting and cyclostationary noise. The developed methodology and expressions can be used to analyze, optimize and build new VCO topologies. A novel topology with an optimized gate to source (GS) feedback is proposed. It exhibits a figure of merit (FOM) better than -190 $\mathrm{dBc} / \mathrm{Hz} / \mathrm{mW}$ for all the frequency offsets.
\end{abstract}

Index Terms - Voltage-controlled oscillator (VCO), Colpitts, phase noise, flicker noise, thermal noise.

\section{INTRODUCTION}

The Cross-coupled CMOS LC voltage controlled oscillators (VCO) have become the most widely used topologies in RF design thanks to their exceptional noise performances [1-2]. However, the emerging communication technologies (mainly OFDM-based broadband standards) and multi-standard requirements impose severe constraints in terms of frequency precision, tuning range and near carrier phase noise [2]. On the other hand, the VCO design difficulties are exacerbated by the technology and voltage scaling. In this context, looking for new VCO alternatives is still a crucial challenge that motivates significant research activities.

Recently, differential Colpitts topologies have attracted a considerable interest $[3,4]$ due to their claimed boostedtransconductance $\left(\mathrm{G}_{\mathrm{m}}\right)$ and superior cyclostationary noise properties compared to conventional single-ended Colpitts and cross-coupled VCOs. The mechanisms governing their phase noise performance are, however, still misunderstood. In fact, the single-ended Colpitts VCO suffers from a poor $\mathrm{G}_{\mathrm{m}}$ and thus, requires higher current to compensate the loss in the tank and sustain the oscillations. Differential Colpitts topologies with a positive cross-coupling feedback [3, 4] (Fig. 1 (DS-Clpt)) have been introduced to boost the $G_{m}$ and thus to relax start-up conditions. However, this topology exacerbates also the nonlinearity of the current. In spite of their importance, the tradeoffs between the superior cyclostationary noise characteristic and the deterioration of the $G_{m}$ by the nonlinearity have never been discussed. The purpose of this paper is to provide a deep understanding of the $\mathrm{G}_{\mathrm{m}}$-boosting, cyclostationary noise

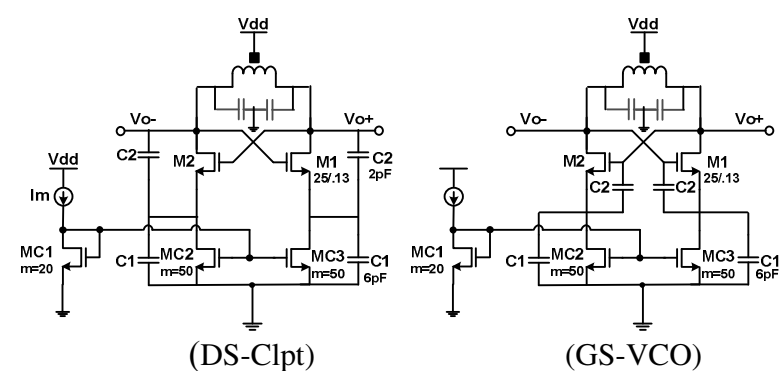

Fig. 1 Differential Colpitts (DS-Clpt) and the proposed alternate $(\mathrm{GS}-\mathrm{VCO})$ designed for $\mathrm{V}_{\mathrm{dd}}=0.7 \mathrm{~V}$ and $\mathrm{I}_{\text {core }}=5, \mathrm{Im}=1 \mathrm{~mA}$.

mechanisms in Colpitts. It will be shown how the class-C configuration of the Colpitts impacts both the low and high offset frequencies phase noise. Furthermore, the detrimental effects of this configuration on the linearity and $\mathrm{G}_{\mathrm{m}}$ will be demonstrated and an alternative topology will be proposed.

\section{GM-Boosting DS-COLPITTS}

The analysis starts with the drain to source differential Colpitts (DS-VCO) shown in Fig. 1 (a) [4]. In this topology, the capacitors $\mathrm{C} 1$, play a major role in the oscillation. Principally, these capacitors give the fundamental and the harmonics of the current the necessary paths to circulate. In contrast, the role of $\mathrm{C} 2$ is quit ambiguous. In the case of single-ended Colpitts, C2 provides the necessary positive feedback for oscillation. In a differential Colpitts however, the positive feedback is already done by cross-coupling the two switching transistors of the VCO.

In order to analyze the effect of this double positive feedback exerted by $\mathrm{C} 2$ and by the switching transistors, we propose to separate their contributions. Using the one

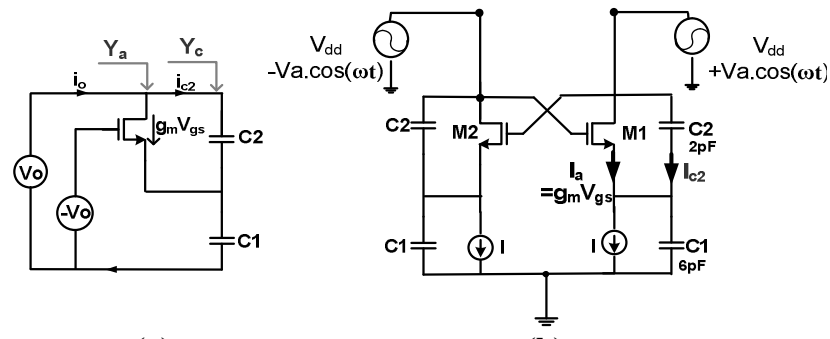

(a)

(b)

Fig. 2 (a) One-port small signal model of DS-VCO (b) the circuit used for large signal analysis of its active part. 
port representation of the active part (Fig. 2 (a)), the input admittance $Y_{\text {in }}$ which is the sum of the active part admittance $Y_{a}$ and the capacitive network admittance $Y_{c}$, can be written as

$$
Y_{i n}=\frac{g_{m} V_{g s}}{V_{o}}+\frac{i_{C 2}}{V_{o}}=\underbrace{\frac{-g_{m} s\left(C_{1}+2 C_{2}\right)}{g_{m}+s\left(C_{1}+C_{2}\right)}}_{Y_{a}}+\underbrace{\frac{2 g_{m} s C_{2}+s^{2} C_{1} C_{2}}{g_{m}+s\left(C_{1}+C_{2}\right)}}_{Y_{c}}
$$

where $g_{m}$ is the transconductance of the switching transistor (M1). In the same way, the total transconductance of the active part $\left(\mathrm{G}_{\mathrm{m}, \mathrm{DS}}\right)$ can be expressed in terms of the real part of $\mathrm{Y}_{\mathrm{a}}$ and the real part of $\mathrm{Y}_{\mathrm{c}}$ as

$$
G_{m, D S}=\operatorname{Re}\left[Y_{a}\right]+\operatorname{Re}\left[Y_{c}\right]
$$

with

$$
\operatorname{Re}\left[Y_{a}\right]=\frac{-g_{m} \omega^{2}\left(C_{1}+2 C_{2}\right)\left(C_{1}+C_{2}\right)}{g_{m}^{2}+\omega^{2}\left(C_{1}+C_{2}\right)^{2}} \approx-g_{m}\left(1+\frac{1}{N+1}\right)
$$

and

$$
\operatorname{Re}\left[Y_{c}\right]=\frac{g_{m} \omega^{2} C_{2}\left(C_{1}+2 C_{2}\right)}{g_{m}^{2}+\omega^{2}\left(C_{1}+C_{2}\right)^{2}} \approx g_{m}\left(\frac{N+2}{(N+1)^{2}}\right)
$$

$\operatorname{Re}\left[\mathrm{Y}_{\mathrm{a}}\right]$ and $\operatorname{Re}\left[\mathrm{Y}_{\mathrm{c}}\right]$ are simplified and expressed versus $\mathrm{N}(\mathrm{N}=\mathrm{C} 1 / \mathrm{C} 2)$ in (3) and (4) by considering the fact that $\mathrm{C}$ is in general very high compared to $\mathrm{g}_{\mathrm{m}}$.

These equations illustrate the antagonistic roles of the capacitive network. On the one hand, it provides an extra positive feedback and thus boosts the negative $G_{m}$ as shown by (3) $\left(\cdot \operatorname{Re}\left[\mathrm{Y}_{\mathrm{a}}\right] \bullet>\mathrm{g}_{\mathrm{m}}\right)$. On the other hand, this capacitive network attenuates the negative $\mathrm{G}_{\mathrm{m}}$ by introducing the positive term described in (4). These two mechanisms, however, would cancel each other if $\mathrm{C} 2$ is chosen small enough compared to $\mathrm{C} 1$. In fact, by replacing (3) and (4) in (2) we can write

$$
G_{m, D S}=-\frac{g_{m} \omega^{2} C_{l}\left(C_{1}+2 C_{2}\right)}{g_{m}^{2}+\omega^{2}\left(C_{1}+C_{2}\right)^{2}} \approx-g_{m}\left(1-(N+1)^{-2}\right) \approx-g_{m}
$$

Since the VCO is fundamentally a large signal circuit, Periodic Study State large-signal analysis (PSS of SpectreRF) can be applied to the circuits of Fig. 2 (b) in order to simulate the currents flowing into the drain of the transistor M1 ( $\left.i_{a}\right)$ and the capacitor C2 $\left(i_{c 2}\right)$ versus the amplitude $\mathrm{V}_{\mathrm{a}}$. The results are used to evaluate $\mathrm{g}_{\mathrm{m}}$ and $\mathrm{G}_{\mathrm{m}, \mathrm{DS}}$ and thus, to verify their equality. The simulated transconductance of the switching transistor $g_{m}$ (i.e. $G_{m, D S}$ when $\mathrm{C} 2=0$ ) is shown in Fig. 3 (continuous line). As expected, its absolute value decreases when the amplitude is sufficiently high to push the transistors in deep triode region.

Now, If we suppose, that $\mathrm{g}_{\mathrm{m}}$ did not change when we introduce the capacitor $\mathrm{C} 2$, we can replace $\mathrm{g}_{\mathrm{m}}$ in (5) by its simulated values (i.e. the continuous line curve of Fig. 3) and calculate the expected theoretical values of $\mathrm{G}_{\mathrm{m}, \mathrm{DS}}$. The results, for $\mathrm{C} 2 / \mathrm{C} 1$ equal to $2 \mathrm{pF} / 6 \mathrm{pF}$, are reported in Fig. 2 (dashed line). As expected, the calculated $\mathrm{G}_{\mathrm{m}, \mathrm{Ds}}$ is practically equal to $\mathrm{g}_{\mathrm{m}}$. This is the main reason why usually, the expression " $\mathrm{G}_{\mathrm{m}}$-boosted topology" is used to qualify the differential Colpitts compared to the single ended one $[3,4]$. Obviously, in comparison to conventional

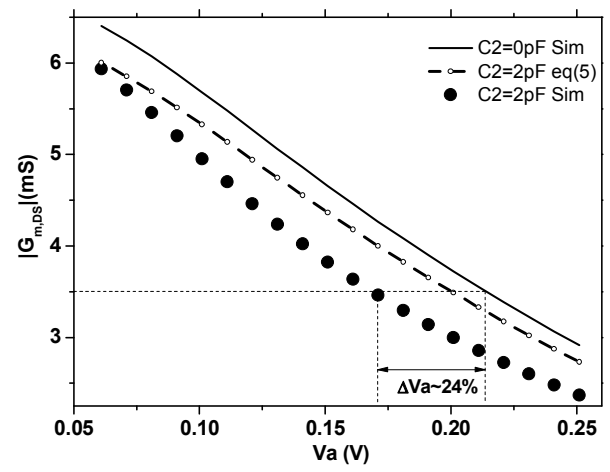

Fig. 3 Simulated and calculated $\mathrm{G}_{\mathrm{m}, \mathrm{DS}}$ versus the amplitude of the signal $\left(\mathrm{V}_{\mathrm{a}}\right)$ with and without $\mathrm{C} 2$.

cross-coupled VCOs (i.e. $\mathrm{g}_{\mathrm{m}}$ ), this transconductance is, at best, similar.

The $\mathrm{G}_{\mathrm{m}, \mathrm{DS}}$ can also be directly evaluated using "PSS" simulation applied to the circuit shown in Fig. 2 (b), through the simulation of $\operatorname{Re}\left[\mathrm{I}_{\mathrm{a}} / \mathrm{V}_{\mathrm{a}}\right]$ and $\left.\operatorname{Re}\left[\mathrm{I}_{\mathrm{c} 2} / \mathrm{V}_{\mathrm{a}}\right]\right)$. The results are shown by the dotted curve in Fig. 3. The degradation of the transconductance is surprisingly well beyond what was expected from the theoretical analysis. The reason of this discrepancy is due to the fact that we assumed in the theoretical analysis that the presence of $\mathrm{C} 2$ has no effect on the transconductance $\left(\mathrm{g}_{\mathrm{m}}\right)$ of the switching transistors, which is a wrong assumption.

The positive feedback exerted by $\mathrm{C} 2$ makes, indeed, the variation of the gate to source voltage $\left(\mathrm{V}_{\mathrm{gs}}\right)$ of the

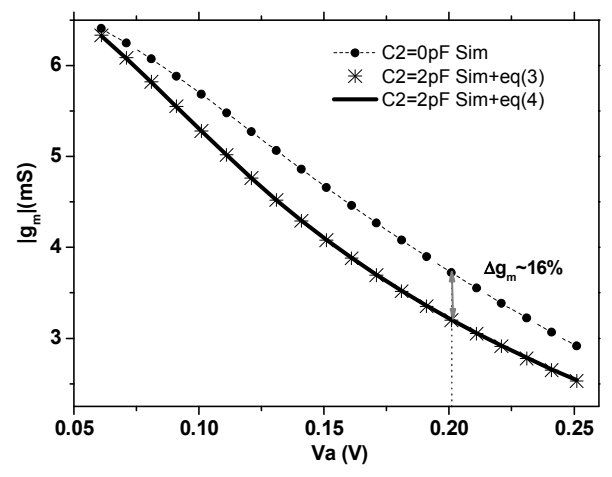

Fig. 4 Variation of $g_{m}$ versus $V_{a}$ for $\mathrm{C} 2$ equal to 0 and $2 \mathrm{pF}$ 
switching transistors more abrupt than in a conventional cross-coupled VCO. Consequently, their drain currents present narrow pulses and thus, a higher non-linearity. Beyond a certain limit, the increases of $\mathrm{V}_{\mathrm{gs}}$ will participate more to increase the second and third harmonics than the fundamental of the drain current, which automatically degrades the $g_{m}$.

The variation of $g_{m}$ versus $V_{a}$ for different values of $\mathrm{C} 2$ can be determined using the relations (3), (4) and the simulated $\operatorname{Re}\left[\mathrm{Y}_{\mathrm{a}}\right]$ and $\operatorname{Re}\left[\mathrm{Y}_{\mathrm{c}}\right]$. The results for $\mathrm{C} 2$ equal 0 and $2 \mathrm{pF}$ are shown in Fig. 4 and confirm the degradation of $g_{m}$ in the presence of $C 2$. Note that the values of $g_{m}$ extracted from (3) and (4) are exactly the same; which proves the consistency of the method and the equations for large signal analysis.

\section{A TRUe GM-Boosted VCO}

Now that the $G_{m}$ degradation of the differential Colpitts is demonstrated, we propose to study a modified topology with a true Gm-boosting capability. This VCO is shown in Fig.1 GS-VCO. It is designed to provide a reverse situation compared to DS-Clpt, in the sense that C2 is inserted between the gate and the source, thereby exerting a negative feedback.

Using the one port representation of the active part (circuit (a) in Fig. 5), the new input admittance $\mathrm{Y}^{\prime}{ }_{\text {in }}$ can be written as

$Y_{i n}^{\prime}=\frac{g_{m} V_{g s}}{V_{o}}+\frac{i_{C 2}}{V_{o}}=\underbrace{\frac{-g_{m} s C_{1}}{g_{m}+s\left(C_{1}+C_{2}\right)}}_{Y_{a}^{\prime}}+\underbrace{\frac{s^{2} C_{1} C_{2}}{g_{m}+s\left(C_{1}+C_{2}\right)}}_{Y_{c}^{\prime}}$

The transconductance $\left(\mathrm{G}_{\mathrm{m}, \mathrm{GS}}\right)$ can be written as

$$
G_{m, G S}=\operatorname{Re}\left[Y_{a}^{\prime}\right]+\operatorname{Re}\left[Y_{c}^{\prime}\right]
$$

with

$$
\operatorname{Re}\left[Y_{a}^{\prime}\right]=\frac{-g_{m} \omega^{2} C_{1}\left(C_{1}+C_{2}\right)}{g_{m}^{2}+\omega^{2}\left(C_{1}+C_{2}\right)^{2}} \approx-g_{m} \frac{N}{N+1}
$$

and

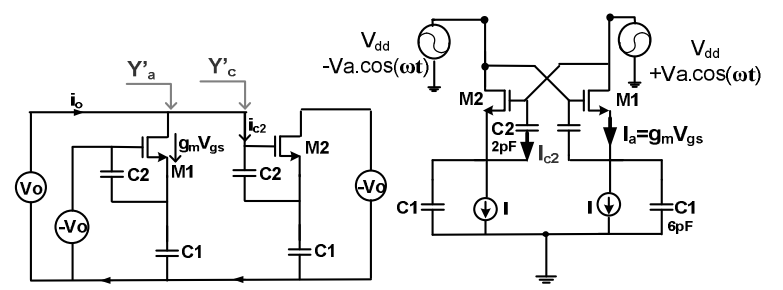

(a)

(b)

Fig. 5 (a) One-port small signal model of GS-VCO, (b) the circuit used for large signal analysis of its active part

$$
\operatorname{Re}\left[Y_{c}^{\prime}\right]=\frac{-g_{m} \omega^{2} C_{2} C_{1}}{g_{m}^{2}+\omega^{2}\left(C_{1}+C_{2}\right)^{2}} \approx-g_{m} \frac{N}{(N+1)^{2}}
$$

These equations illustrate the fact that the role of $\mathrm{C} 2$ is reversed in comparison to the DS-Colpitts. On the one hand, it provides a negative feedback and thus attenuate the negative $G_{m}$ as shown by $(8)(N /(N+1)<1)$. On the other hand, this capacitive network boost the negative $G_{m}$ by introducing the negative term described in (9).

Fig. 6 (a) shows the simulated $\mathrm{G}_{\mathrm{m}, \mathrm{GS}}$ versus $\mathrm{V}_{\mathrm{a}}$ for $\mathrm{C} 2$ equal $2 \mathrm{pF}$ (continuous line) using PSS applied to the circuit (b) of Fig. 5. In contrast to DS-Clpt case, $\mathrm{G}_{\mathrm{m}, \mathrm{GS}}$ is not deteriorated but boosted in the presence of $\mathrm{C} 2$. In fact, the negative feedback exerted by $\mathrm{C} 2$ results in a smoother variation of the gate to source voltage $\left(\mathrm{V}_{\mathrm{gs}}\right)$ and thus, a more linear drain current. Consequently, $\mathrm{g}_{\mathrm{m}}$ of the switching transistors is boosted in this topology. The $\mathrm{G}_{\mathrm{m}}$ boosting in GS-VCO constitutes a crucial advantage for the signal strength. For instance, if the loss in the tank results in a parallel resistance of $285 \Omega$ (i.e. $1 / 3.5 \mathrm{mS}$ ), the amplitude, required to compensate this loss, will be equal to $171 \mathrm{mV}$ for DS-Clpt. The same configuration will results $231 \mathrm{mV}$ for GS-Clpt, which constitutes an improvement of $35 \%$.

The variation of $g_{m}$ versus $V_{a}$ determined using the

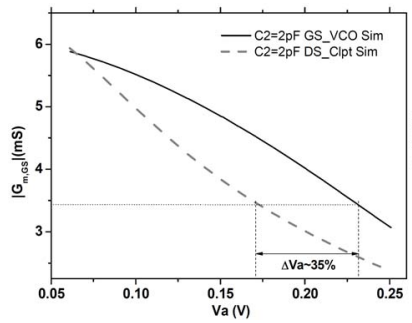

(a)

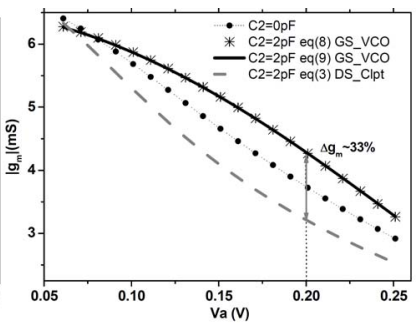

(b)
Fig. 6 Simulated $\mathrm{G}_{\mathrm{m}, \mathrm{GS}}$ (a) and $\mathrm{g}_{\mathrm{m}}$ (b) of GS-VCO. $\mathrm{G}_{\mathrm{m}, \mathrm{GS}}$ and $\mathrm{g}_{\mathrm{m}}$ of DS-Clpt is also shown for comparison

relations (8), (9) and the simulated $\operatorname{Re}\left[\mathrm{Y}_{\mathrm{a}}\right]$ and $\operatorname{Re}\left[\mathrm{Y}_{\mathrm{c}}\right]$ are shown in Fig. 6 (b) (continuous curve). The results confirm the $\mathrm{g}_{\mathrm{m}}$-boosting by the $\mathrm{C} 2$ negative feedback.

\section{CyClostationary NOISE: A COMPARATIVE STUDY}

The gm degradation in DS-Clpt is due to the same mechanism that improves the cyclostationary noise properties of the VCO i.e. a near class-C configuration. Fig. 7 shows the simulated drain currents and output voltage for DS-Clpt and GS-VCO. The results confirm the superior cyclostationary noise properties of DS-Clpt and the superior linearity of GS-VCO. When the current exhibits narrow pulses that coincide with the voltage 
minimums, as for DS-Clpt, the noise sources perturb mainly the amplitude of the signal and thus, would generate less phase noise $[3,4]$. On the other hand, the deterioration of the VCO transconductance due to the current nonlinearity would have a direct detrimental effect on phase noise through the diminution of the amplitude of the signal since the phase noise is, indeed, a noise to signal ratio [5]. Moreover, the drops in the voltage swing will modify the AM to PM conversion. This ambivalent effect reveals the tradeoff between the improvement of the cyclostationary noise properties and resulting $\mathrm{g}_{\mathrm{m}}$ degradation.

In order to decide between this two design strategies, a

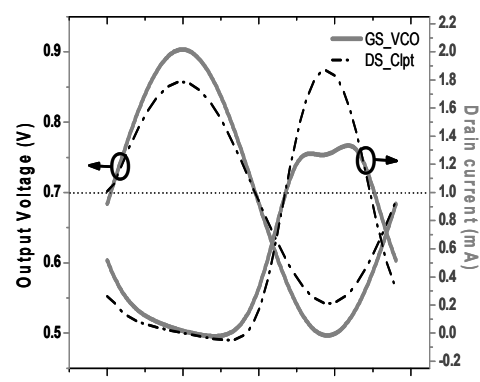

(a)

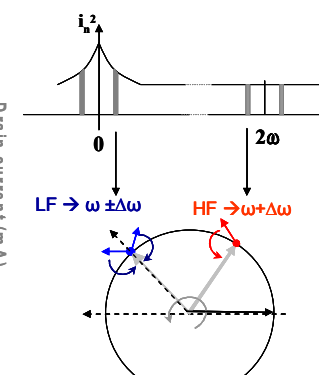

(b)
Fig. 7 (a) Variation of the drain current and voltage for DS-Clpt and GS-VCO, (b) a diagram showing the phase and amplitude perturbation of the VCO signal by low and high frequency noise.

DS-Clpt and GS-VCO were designed in CMOS $90 \mathrm{~nm}$ technology and their noise performance analyzed. The size of the components in the two designs are exactly the same (i.e. $\mathrm{W} / \mathrm{L}=250 \mathrm{~nm} / 130 \mathrm{~nm}, \mathrm{~L}=1.6 \mathrm{nH}, \mathrm{Q}($ at $3 \mathrm{GHz}) \approx$ 15 , and $\mathrm{V}_{\mathrm{DD}}=0.7 \mathrm{~V}$ ). Fig. 8 shows the phase noise versus frequency of the two VCOs. As illustrated, GS-VCO exhibits a better close-in phase noise $(10 \mathrm{dBc} / \mathrm{Hz}$ lower at $1 \mathrm{KHz}$ ). Beyond $100 \mathrm{KHz}$, the noise performances of the

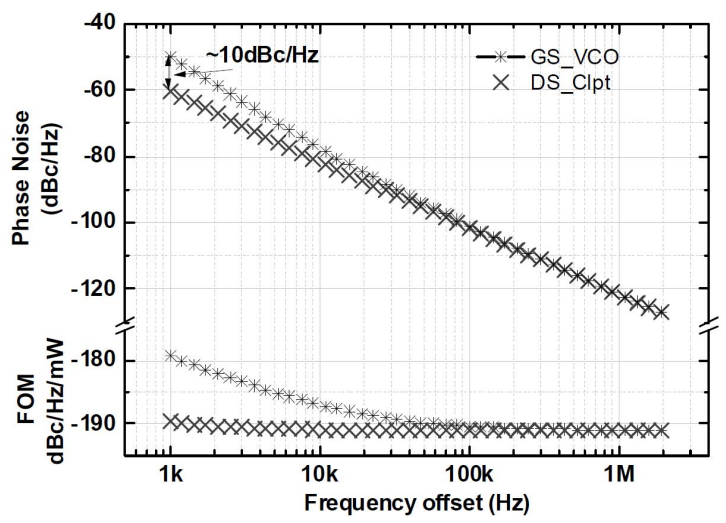

Fig. 8 Phase noise and FOM of DS-Clpt and GS-VCO. two VCOs are quite similar. These results confirm the useless of the class-C configuration in the close-in phase noise improvement. In fact, as explained in Fig. 7 (b), the low frequency noise at $\pm \Delta \omega$ (e.g. MOS flicker noise) generates, at any way, only an amplitude noise after upconversion to $\omega \pm \Delta \omega[1]$, since the negative $(-\Delta \omega)$ and positive parts $(+\Delta \omega)$ of their spectrum are correlated. The close-in phase noise is thus generated mainly through AM to PM conversion [5]. Consequently, the fact that the noise current perturbs mainly the amplitude of the signal, did not change anything to the up-converted phase noise. Increasing the amplitude through a true Gm-boosting technique as in GS-VCO is therefore more effective. For the high frequency noise at $2 \omega \pm \Delta \omega$, the negative and positive parts of the spectrum are not correlated (Fig. 7 (b)) and thus, generate phase and amplitude noise after down conversion to $\omega+\Delta \omega$ [1]. The class- $C$ configuration is more effective in this case, even if, in general, it will enables only to compensate the $G_{m}$ degradation effect (Fig. 8).

\section{CONCLUSION}

A comprehensive analysis of $\mathrm{G}_{\mathrm{m}}$ and the phase noise in differential Colpitts was presented. The useless of the class-C configuration in close-in phase noise improvement and the $\mathrm{Gm}$ degradation by the nonlinearities are demonstrated. An enhanced VCO topology with a true $\mathrm{Gm}$-boosting capacitive feedback is proposed. It exhibits an $\mathrm{FOM}$ better than $-190 \mathrm{dBc} / \mathrm{Hz} / \mathrm{mW}$ for all the frequency offsets and outperforms the conventional Colpitts by several $\mathrm{dB}$ in the flicker noise region.

\section{REFERENCES}

[1] J. J. Rael and A. A. Abidi, "Physical processes of phase noise in differential LC oscillator," Proc. IEEE Custom Integrated Circuits Conf. (CICC), Sep. 2000, pp. 569-571.

[2] A. Koukab, Y. Lei, and M. Declercq "A GSM-GPRS / UMTS FDDTDD/ WLAN 802.11 a-b-g Multi-Standard Carrier Generation System" IEEE J. Solid-State Circuits, vol. 41, no. 7, pp. 1513 - 1521, Jul. 2006.

[3] R. Aparicio and A. Hajimiri, "A noise-shifting differential Colpitts VCO," IEEE J. Solid-State Circuits, vol. 37, no. 12, pp. 1728-1736, Dec. 2002.

[4] X. Li, S. Shekhar, and D. J. Allstot, "Gm -boosted commongate LNA and differential Colpitts VCO/QVCO in 0.18- $\mathrm{m}$ CMOS," IEEE J. Solid-State Circuits, vol. 40, no. 12, pp. 2609-2619, Dec. 2005

[5] A. Koukab, "LC-VCO Design With Dual-Gm,Boosted for RF Oscillation and Attenuated for LF Noise" IEEE Micow. Wireless Compon. Lett., vol. 20, no. 12, pp. 675-677, Dec. 2010. 